\title{
Using a Phenomenological Mathematical Model to Reproduce the Interaction of Endogenous and Exogenous Oscillations under Neurocontrol
}

DOI: $10.17691 / \mathrm{stm} 2019.11 .1 .12$

Received October 14, 2018

I.V. Nuidel, PhD, Senior Researcher ${ }^{1}$; Senior Researcher, Laboratory of Advanced Methods

for Multidimensional Data Analysis, Institute of Information Technology, Mathematics, and Mechanics;

A.V. Kolosov, Student';

V.A. Demareva, PhD, Assistant, Department of Psychophysiology2;

V.G. Yakhno, DSc, Head of the Laboratory of Autowave Processes ${ }^{1}$; Professor, Department of Neurotechnology, Institute of Biology and Biomedicine ${ }^{2}$

${ }^{1}$ Federal Research Center Institute of Applied Physics, Russian Academy of Sciences, 46 Ulyanova St., Nizhny Novgorod, 603950, Russia;

${ }^{2}$ National Research Lobachevsky State University of Nizhny Novgorod, 23 Prospekt Gagarina, Nizhny Novgorod, 603950, Russia

The aim of the study was to evaluate the possibility of using a phenomenological mathematical model of the thalamocortical unit cell to describe the frequency-time responses of the real thalamocortical system (namely, various alpha rhythm modulations) and reproduce the signal dynamics in the process of neurobiocontrol.

Materials and Methods. The experimental part of this study (the resonant neurobiocontrol with double feedback based on the BioFeedBack2 software-hardware complex) was carried out according to the hybrid protocol: background - before/after: 2-minute record of the baseline vertex EEG (the active electrode $-\mathrm{Cz}$ grounding and the reference electrodes held on the earlobes); frequency scan during $210 \mathrm{~s}$ : exposure to pulsed infrared radiation with an increasing rate of from 8 to $14 \mathrm{~Hz}$ (frequency step $-0.1 \mathrm{~Hz}$, time step - $3 \mathrm{~s}$ ) and a music-like sound signal, the tone and volume of which determined by the peak amplitude in the spectrum of the current EEG in the range of $8-14 \mathrm{~Hz}$. The characteristic feedback time is $10 \mathrm{~ms}$, the frequency accuracy is $0.2-0.4 \mathrm{~Hz}$. Periodic noise pulses, presented with a frequency related to the baseline heart rate, have been added to the sound signal.

For the calculations, a previously developed phenomenological model of the thalamocortical unit cell was used. The model incorporates interacting modules simulating the major neuronal modules of the brain, i.e., the thalamus, the cortex and the thalamic reticular nuclei.

Results and Discussion. Using the proposed phenomenological mathematical model of the thalamocortical unit cell, we obtained frequency-time responses of the model signal, which reproduces the frequency pattern of the real EEG signal. The model simulates the situation when the baseline alpha rhythm changes in response to an external factor with the known thalamocortical parameters. In the future, this information will improve the current feedback procedures of biocontrol aimed at enhancing the cognitive power of the brain. Appropriate training will allow controlling the alpha rhythm frequency (neurobiolcontrol) in such a way that, by the objective psychophysical criteria, the subjects will have their cognitive activity enhanced, and, by the subjective assessment, their well-being will improve.

Conclusion. In this study, a neuro-informational approach to personalized brain rhythm management is demonstrated. It is now possible to reproduce individual features of a complex information processing system using the proposed phenomenological model of the thalamocortical unit cell. system.

Key words: neuronal interface; electroencephalogram; EEG; neurobiocontrol; brain rhythms; mathematical model; thalamocortical

\section{Introduction}

A major task of today is a personalized approach to research, medical diagnostics, and provision of medical care $[1,2]$. In this respect, it is necessary to improve the simulators of complex systems capable of demonstrating mutual connections, direct and inverse, between the diagnostic information (e.g., EEG or ECG signals) and the patient's psychophysical markers (objective, obtained by measurements, and subjective, obtained by questionnaires). The "brain-computer interface" [3] and "neurobiocontrol" technologies are aimed at achieving precisely this goal. These technologies are based on modulating the brain activity by using feedback signals from brain biopotentials, in particular from the patient's own rhythmic processes, namely the EEG (in other words, neurobiocontrol) [4, 5]. To simulate complex systems for signal processing in humans, phenomenological models are used.

The aim of the study was to evaluate the possibility of using a phenomenological mathematical model of the thalamocortical unit cell to describe the frequency-time

Corresponding author: Irina V. Nuidel, e-mail: nuidel@appl.sci-nnov.ru 
responses of the real thalamocortical system (namely, various alpha rhythm modulations) and reproduce the signal dynamics in the process of neurobiocontrol.

We also aimed to identify how the signal is transformed after processing in the thalamocortical system and what happens when a frequency-modulated signal with a linearly increasing frequency is fed into the system.

\section{Materials and Methods}

Neuronal modules in the phenomenological model of the thalamocortical system. The results of experimental neurophysiological studies indicate that interconnected neuronal modules (the cortex, the reticular nuclei of the thalamus, and the specific thalamus) play an important role in the information processing. The interacting links of the thalamocortical chain determine the architecture of the phenomenological model of the thalamocortical unit cell.

In reality, a single neuronal module includes pyramidal neurons, inhibitory cortex interneurons, specific thalamic nuclei neurons, and inhibitory neurons of the thalamic reticular nucleus, which are all interconnected. The inter-module interaction is schematically shown in Figure 1 [6]. The thalamus is a complex polyfunctional formation that includes relay nuclei, where the afferent signals from the sensory organs are transmitted to the respective areas of the cerebral cortex. In other words, in specific (sensory or relay) nuclei of the thalamus, sensory information from axons of the ascending afferent pathways undergoes synaptic switching to the next, final neurons that have their processes in the

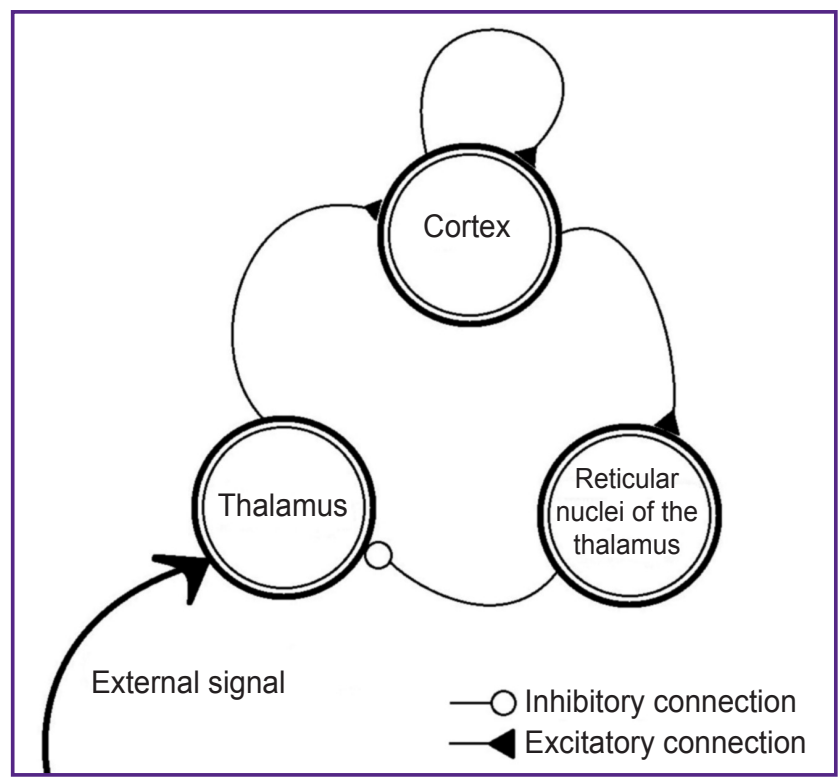

Figure 1. Scheme of functional connections between the subsystems in the thalamocortical unit cell [6]

The arrow at the bottom indicates the sensory input into the thalamus respective sensory projection areas of the cerebral cortex. For example, the lateral geniculate body of the brain represents a specific nucleus of the visual sensory system, which has direct connections with the occipital (visual) projection areas of the cerebral cortex [6].

Under normal physiological conditions, thalamocortical system functions as follows: 1) the external signal first activates neurons in the specific (relay) thalamic nucleus; 2) along the thalamocortical pathway, the excitation reaches the cortex, where the cortical pyramidal neurons are connected with inhibitory interneurons, which can in some way modulate their activity; 3 ) the further spread of excitation occurs along the descending corticothalamic pathways to the specific and reticular nuclei of the thalamus; 4) the latter is connected to the relay nucleus of the thalamus via the inhibitory pathways and, therefore, can interrupt the excitation arriving from the specific thalamic nucleus to the cortex.

The reticular nucleus of the thalamus is a kind of gateway for sensory information entering the cortex. It has no direct access to the cortex, but it does receive input signals from the cortex and thalamic nuclei and, apparently, functions as an intrathalamic control center.

The inhibitory action of the thalamic reticular nuclei suppresses the "strobe-like" activity, during which, for example, characteristics of the input sensory signal in the cortex are identified; then followed a period of inactivity. As a result, after the input signal passes through the thalamocortical system, a discontinuous strobed representation of this sensory signal is stored in the cortex.

The present study addressed the focused model of the thalamocortical function without considering structures of the internal modules, i.e., the specific thalamic nucleus; the inhibitory reticular nonspecific thalamic nucleus, or the cortex areas associated with the thalamic nuclei [7].

For the calculations, a previously developed phenomenological model of the elemental thalamocortical unit cell was used [7]. This model incorporates the interacting modules that simulate such neuronal modules of the brain as the thalamus, cortex and reticular nuclei of the thalamus. This phenomenological model (schematized in Figure 1) is represented by a system of differential equations reflecting the external signal processing between the thalamus, cortex and reticular thalamic nuclei:

$$
\begin{gathered}
\frac{\mathrm{d} U_{1}}{\mathrm{~d} t}=-\frac{U_{1}}{\tau_{1}}+k_{1} F_{1}\left[-T_{1}+k_{e x} U_{e x}-k_{13} U_{3}\right] ; \\
\frac{\mathrm{d} U_{2}}{\mathrm{~d} t}=-\frac{U_{2}}{\tau_{2}}+k_{2} F_{2}\left[-T_{2}+k_{21} U_{1}+k_{22} U_{2}\right] ; \\
\frac{\mathrm{d} U_{3}}{\mathrm{~d} t}=-\frac{U_{3}}{\tau_{3}}+k_{3} F_{3}\left[-T_{3}+k_{32} U_{2}\right],
\end{gathered}
$$

where $U_{1}, U_{2}, U_{3}$ - the averaged neuronal activity 
in selected areas of the thalamus, cortex, and reticular thalamic nuclei, respectively; $\tau_{i}-$ the characteristic time of activity attenuation in the respective neuronal ensembles; $k_{i}$ - the pulsed activity amplitude in the respective neuronal ensembles; $T_{i}$ - the averaged values of the excitation thresholds for the respective neuronal ensembles; $U_{e x}-$ the input signal entering the thalamus; $k_{i j}$ - the coefficients of interconnection between subsystems in the thalamocortical unit cell; $F_{i}[]$ - step-shaped functions, the steepness of which characterizes the scatter of threshold values around their mean in the ensemble of interest (another name: the power supply functions); inside the square brackets is the analog of the membrane postsynaptic potential in the respective neuronal ensembles; $i=1,2,3$.

Since all external inputs in the cortex are associated with thalamic structures, this model is used to process the signals and their spectra in the case of frequency modulation of the thalamic signal by an external signal with a linearly increasing frequency.

The input sensory signal is a frequencymodulated signal: in the psychophysiological experiment, it is equivalent to an infrared highfrequency modulated signal with respect to the EEG signal own frequency. The signal from the variable cortex is similar to the integrated bioelectric EEG signal.

Neurobiocontrol protocol. This dual feedback resonant neurobiocontrol is based on the BioFeedBack2 software-hardware setup and run according to the following hybrid protocol: background - before/after: 2-minute record of the baseline vertex EEG (the active electrode $-\mathrm{Cz}$ grounding and the reference electrodes held on the earlobes); frequency scan during $210 \mathrm{~s}$ : exposure to pulsed infrared radiation with an increasing frequency from 8 to $14 \mathrm{~Hz}$ (frequency step $-0.1 \mathrm{~Hz}$, time step - $3 \mathrm{~s}$ ) and a music-like sound signal, the tone and volume of which determined by the peak amplitude in the spectrum of the current EEG in the range of 8-14 Hz. The characteristic feedback time is $10 \mathrm{~ms}$, the frequency accuracy is $0.2-0.4 \mathrm{~Hz}$. Periodic noise pulses, presented with a frequency related to the baseline heart rate, have been added to the sound signal $[1,5]$.

In the course of the experiment, a pulsating (with a linearly increasing frequency) infrared lamp is directed at the subject's closed eyes. The EEG signal is recorded before exposure to the infrared radiation, during the exposure and after the exposure. Next, the dynamic spectrum of the signal is created.

Usually, the dynamic spectrum of EEG signals of a healthy person looks like that in Figure 2 (b). Specific features for each individual subject include the basic natural frequency of the alpha rhythm in the range from 8 to $12 \mathrm{~Hz}$ and the width of the fuzzy spectrum around it.

There is evidence that under external exposure, the thalamocortical system functions in the forced oscillations

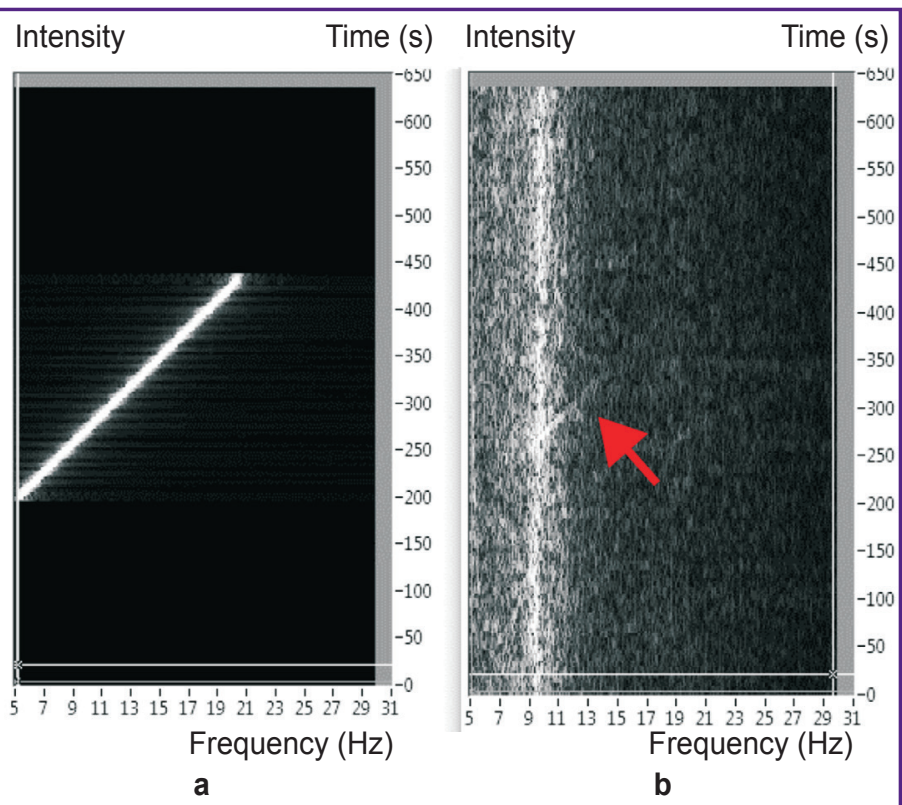

Figure 2. Dynamic spectra of the external infrared signal with a linearly increasing frequency (a) and of the respective thalamocortical EEG of one of the subjects (b)

The red arrow indicates the appearance of responses at a forced requency

mode for some time (Figure 2). This event occurs at the moment when the frequency of the external signal is higher than the natural frequency of the thalamocortical system; this situation exists until the frequency of the external signal becomes so high that the subject perceives it as constant, with the amplitude equal to the mean value of the periodic signal. This dynamic mode corresponds to the forced oscillations (filling) with a selfoscillating envelope; it can be well identified when the external signal is superimposed on the EEG.

\section{Results and Discussion}

In this study, of all the parameters, only the amplitude of the external signal $U_{e x}$ varied. The numerical values of the parameters were: $k_{i}=1, i=1,2,3 ; t_{i}=1, i=1,2,3$; $T_{1}=0 ; \quad T_{2}=0.5 ; \quad T_{3}=0.5 ; \quad k_{e x}=1 ; \quad k_{13}=1 ; \quad k_{21}=1 ; \quad k_{22}=0 ;$ $k_{32}=1$. The smoothly varying power supply function $F_{i}[]=0.5+0.5 \cdot \tanh (20[])$ was used.

For the $U_{e x}$, the signal presented in Figure 3, similar to the experimental one was used; in this signal, the frequency of the oscillating part increased in the linear mode.

The response of the thalamus $\left(U_{1}\right)$ to external signal of this type is shown in Figure 4.

Figures 3 and 4 demonstrate three states of the system: before an exposure to external signal, during exposure to a high-frequency external signal, and after the exposure. All these states are characterized by the natural frequency of the thalamocortical system, which depends on the mean amplitude of the external signal [7]. 


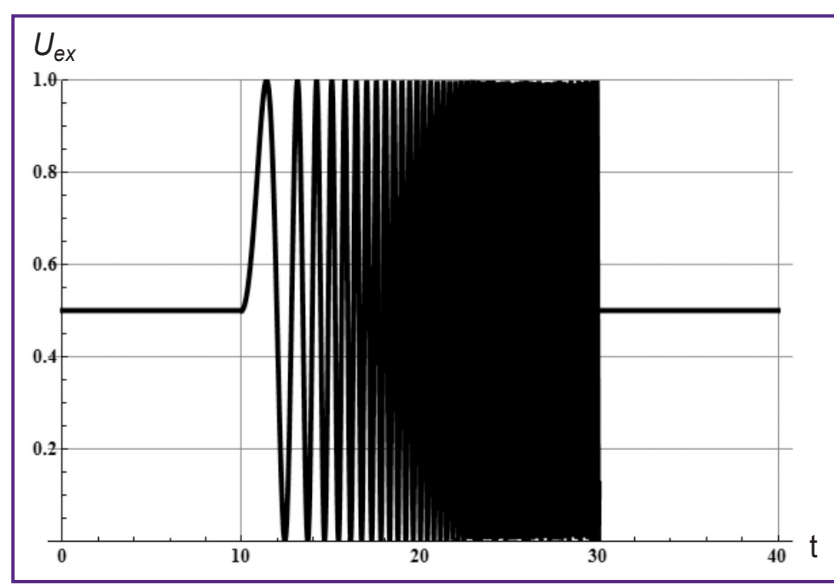

Figure 3. The time dimension of the simulated external signal
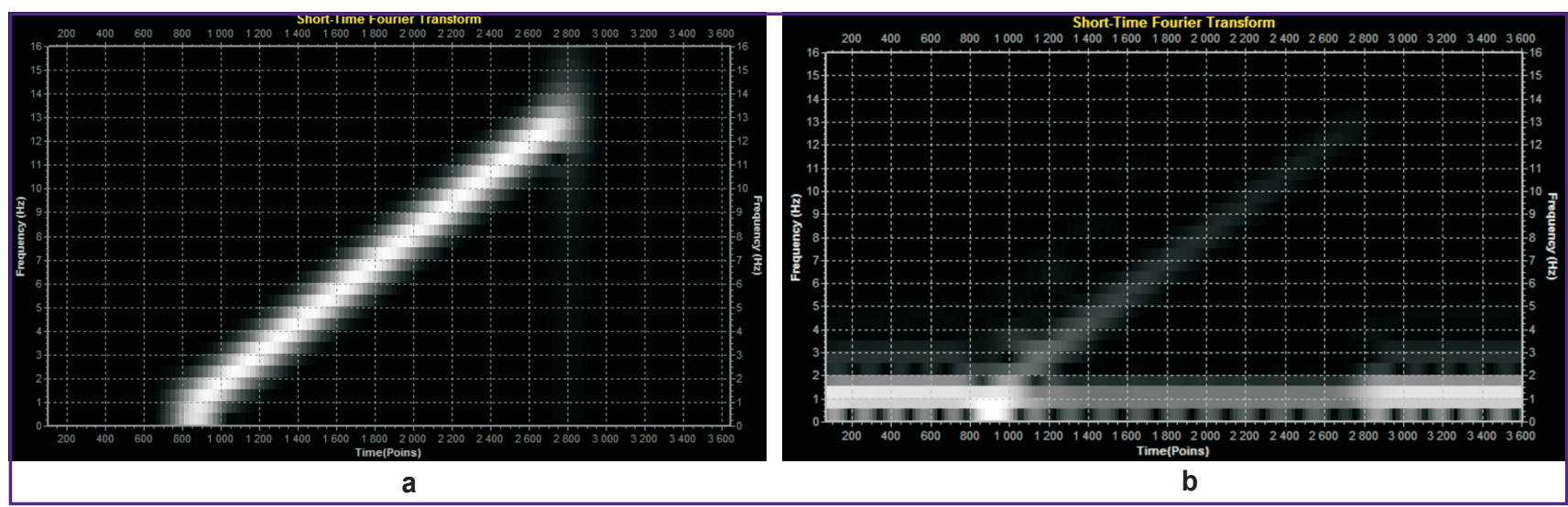

Figure 5. Dynamic spectrum of the external signal $U_{\text {ex }}(\mathrm{a})$ and the thalamic signal $U_{1}(\mathrm{~b})$

Vertical axis: frequency; horizontal axis: time

"High frequency exposure" means that the frequency of the external signal is substantially higher than the natural frequency of the thalamocortical unit cell. Dynamic spectra of both (external and thalamic) signals are shown in Figure 5.

Thus, using the phenomenological mathematical model of the thalamocortical unit cell, we obtained frequency-time responses of a model signal reproducing the frequency pattern of the real EEG signal $[8,9]$. The model simulates a situation when the baseline alpha rhythm changes in response to an external factor with the known thalamocortical parameters. The resulting information provides the opportunity to improve the current feedback procedures of biocontrol aimed to enhance the cognitive activity of the brain. Special training will allow one to control the alpha rhythm frequency (neurobiolcontrol) in such a way that, by the objective psychophysical criteria, the subject will have his/her cognitive activity enhanced, and, by the subjective assessment, his/her well-being will improve.

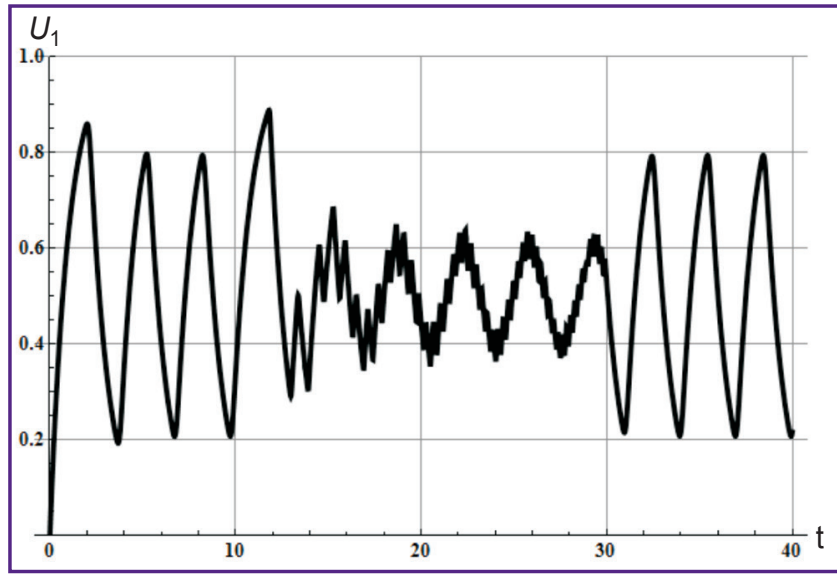

Figure 4. Thalamic response to the external signal with a linearly increasing frequency 


\section{References}

1. Fedotchev A.l., Oh Sang Joon, Bondar' A.T., Semenov V.S. Sovremennye vozmozhnosti $i$ podkhody $k$ aktivizatsii kognitivnoy deyatel'nosti i protsessov obucheniya u cheloveka [Current resources and approaches to activization of cognitive activity and learning processes in humans]. Pushchino: IBK RAN; 2017.

2. Hammond D.C. What is neurofeedback: an update. J Neurother 2011; 15(4): 305-336, https://doi.org/10.1080/108 74208.2011.623090.

3. Miranda R.A., Casebeer W.D., Hein A.M., Judy J.W., Krotkov E.P., Laabs T.L., Manzo J.E., Pankratz K.G., Pratt G.A., Sanchez J.C., Weber D.J., Wheeler T.L., Ling G.S. DARPA-funded efforts in the development of novel braincomputer interface technologies. J Neurosci Methods 2015; 244: 52-67, https://doi.org/10.1016/j.jneumeth.2014.07.019.

4. Fedotchev A.I., Parin S.B., Polevaya S.A., Velikova S.D. Brain-computer interface and neurofeedback technologies: current state, problems and clinical prospects (review). Sovremennye tehnologii v medicine 2017; 9(1): 175, https:// doi.org/10.17691/stm2017.9.1.22.

5. Fedotchev A.I., Bondar' A.T., Bakhchina A.V.,
Parin S.B., Polevaya S.A., Radchenko G.S. Music-acoustic signals controlled by subject's brain potentials in the correction of unfavorable functional states. Uspekhi fiziologicheskikh nauk 2016; 47(1): 69-79.

6. Coulter D.A. Thalamocortical anatomy and physiology. In: Engel J. Jr., Pedley T.A. (editors). Epilepsy: a comprehensive textbook. Philadelphia: Lippincott Williams \& Wilkins; 2007; p. 353-366.

7. Kolosov A.V., Nuidel I.V., Yakhno V.G. Research of dynamic modes in the mathematical model of elementary thalamocortical cell. Izvestiya vysshikh uchebnykh zavedeniy. Prikladnaya nelineynaya dinamika 2016; 24(5): 72-83, https:// doi.org/10.18500/0869-6632-2016-24-5-72-83.

8. Bondar A., Shubina L. Nonlinear reactions of limbic structure electrical activity in response to rhythmical photostimulation in guinea pigs. Brain Res Bull 2018; 143: 7382, https://doi.org/10.1016/j.brainresbull.2018.10.002.

9. Fedotchev A.I., Bondar A.T., Bakhchina A.V., Grigorieva V.N., Katayev A.A., Parin S.B., Radchenko G.S., Polevaya S.A. Transformation of patient's EEG oscillators into music-like signals for correction of stress-induced functional states. Sovremennye tehnologii v medicine 2016; 8(1): 93-98, https://doi.org/10.17691/stm2016.8.1.12. 